\title{
First order periodic problem at resonance with nonlinear impulses
}

\author{
Pavel Drábek ${ }^{1,2}$ and Martina Langerováa ${ }^{*}$
}

\author{
"Correspondence: \\ mlanger@ntis.zcu.cz \\ ${ }^{2}$ NTIS, University of West Bohemia, \\ Univerzitní 22, Plzeň, 306 14, Czech \\ Republic \\ Full list of author information is \\ available at the end of the article
}

\begin{abstract}
We consider the nonlinear first order periodic problem with nonlinear impulses. We apply the Schaeffer fixed point theorem and prove the existence results under Landesman-Lazer type sufficient conditions. We formulate also necessary conditions in some special cases. The impulses can be viewed as a control which compensates the influence of external forces and vice versa.
\end{abstract}

MSC: Primary 34A37; 34B37; secondary 34F15; 47H11

Keywords: impulsive differential equations; Landesman-Lazer conditions; topological degree theory; Schaeffer fixed point theorem

\section{Introduction}

In this paper we deal with a nonlinear boundary value problem of the first order,

$$
\begin{aligned}
& u^{\prime}(t)+a(t) u(t)=f(t, u(t)), \quad t \in[0, T], \\
& u(0)=u(T) .
\end{aligned}
$$

At prescribed points in the interval $(0, T)$ the solution is subject to the impulses which depend on an actual value of the solution. These impulses can be interpreted as a control of the external forces represented by $f$ in order to get existence or nonexistence a periodic solution of (1). Vice versa, the external forces can be interpreted as a control of given impulses.

Impulsive problems have attracted the attention of mathematicians for several decades. Let us mention the classical books [1-4]. The first order boundary value problems with impulses were studied recently in many research articles such as [5-12]. Optimal control of space trajectories with applications to maneuvers of spacecraft or satellite constellation are considered in papers [13-16].

According to the best of our knowledge, this paper is the first one to treat the periodic first order problem at resonance with external forces and impulses satisfying the Landesman-Lazer type conditions (see (3)-(7) below and compare with conditions in [17]). Similar conditions for Dirichlet problems at resonance, but for second order semilinear and quasilinear equations (involving the $p$-Laplacian), are formulated in the authors' papers [18] and [19], respectively. The authors apply the topological methods in [18] and variational methods in [19] to prove the existence results. In this paper we rely on the Schaeffer fixed point theorem, which is based on the topological degree argument.

c 2014 Drábek and Langerová; licensee Springer. This is an Open Access article distributed under the terms of the Creative Commons Attribution License (http://creativecommons.org/licenses/by/4.0), which permits unrestricted use, distribution, and reproduction in any medium, provided the original work is properly credited. 
In order to formulate the main results precisely, we need some notation.

Let $T>0, J=[0, T], 0=t_{0}<t_{1}<t_{2}<\cdots<t_{p}<t_{p+1}=T$ and set $J^{\prime}=J \backslash\left\{t_{1}, t_{2}, \ldots, t_{p}\right\}$. We denote by $B C\left(J^{\prime}\right)$ the Banach space of all bounded and continuous functions defined on $J^{\prime}$ equipped with the usual supremum norm on $J^{\prime}$. We also let

$$
\begin{aligned}
X:= & \left\{u: J \rightarrow \mathbb{R}: u(t) \text { is continuous in } J^{\prime} \text {, one-sided limits } u\left(t_{j}^{+}\right),\right. \\
& \left.u\left(t_{j}^{-}\right) \text {exist that are finite at } t_{j}, u\left(t_{j}\right)=u\left(t_{j}^{-}\right), j=1,2, \ldots, p ; u(0)=u(T)\right\},
\end{aligned}
$$

equipped with the norm

$$
\|u\|=\max _{t \in J}|u(t)|
$$

Then $(X,\|\cdot\|)$ is a Banach space.

We assume that $a \in X, f \in C\left(J^{\prime} \times \mathbb{R}\right)$ is such that $\forall s \in \mathbb{R}, \forall j=1,2, \ldots, p$ the limits

$$
f\left(t_{j}, s\right):=\lim _{t \rightarrow t_{j}^{-}} f(t, s) \text { and } \lim _{t \rightarrow t_{j}^{+}} f(t, s)
$$

exist.

Let $I_{j}: \mathbb{R} \rightarrow \mathbb{R}, I_{j} \in C(\mathbb{R}), j=1,2, \ldots, p$. We consider the following nonlinear impulse conditions:

$$
u\left(t_{j}^{+}\right)=u\left(t_{j}\right)-I_{j}\left(u\left(t_{j}\right)\right), \quad j=1,2, \ldots, p .
$$

By a solution of (1), (2) we understand a function $u \in X$ such that $u^{\prime} \in X$ and (1), (2) hold true.

Our main results concern the resonance case:

$$
\int_{0}^{T} a(\tau) \mathrm{d} \tau=0
$$

We also assume that $f$ is bounded on $J \times \mathbb{R}$ and for $t \in J$ the limits

$$
f_{ \pm}(t):=\lim _{s \rightarrow \pm \infty} f(t, s) \quad \text { and } \quad I_{j}( \pm \infty):=\lim _{s \rightarrow \pm \infty} I_{j}(s), \quad j=1,2, \ldots, p
$$

exist. We admit $I_{j}(-\infty)=-\infty$ and $I_{j}(+\infty)=+\infty$ but we require $I_{j}$ to be sublinear at $\pm \infty$, that is, we assume

$$
\lim _{s \rightarrow \pm \infty} \frac{I_{j}(s)}{s}=0 \quad \text { for } j=1,2, \ldots, p .
$$

In the following theorem the mutual connection among $f_{ \pm}(t)$ and $I_{j}( \pm \infty)$ plays the key role.

Theorem 1 Let the following two inequalities hold:

$$
\int_{0}^{T} f_{+}(t) e^{\int_{0}^{t} a(\tau) \mathrm{d} \tau} \mathrm{d} t<\sum_{j=1}^{p} I_{j}(+\infty) e^{\int_{0}^{t_{j}} a(\tau) \mathrm{d} \tau},
$$




$$
\int_{0}^{T} f_{-}(t) e^{\int_{0}^{t} a(\tau) \mathrm{d} \tau} \mathrm{d} t>\sum_{j=1}^{p} I_{j}(-\infty) e^{\int_{0}^{t_{j}} a(\tau) \mathrm{d} \tau}
$$

or, alternatively,

$$
\begin{aligned}
& \int_{0}^{T} f_{+}(t) e^{\int_{0}^{t} a(\tau) \mathrm{d} \tau} \mathrm{d} t>\sum_{j=1}^{p} I_{j}(+\infty) e^{\int_{0}^{t_{j}} a(\tau) \mathrm{d} \tau}, \\
& \int_{0}^{T} f_{-}(t) e^{\int_{0}^{t} a(\tau) \mathrm{d} \tau} \mathrm{d} t<\sum_{j=1}^{p} I_{j}(-\infty) e^{\int_{0}^{t_{j}} a(\tau) \mathrm{d} \tau} .
\end{aligned}
$$

Then the nonlinear impulsive problem (1), (2) has at least one solution.

In particular, for the linear equation we have the following necessary and sufficient condition.

Corollary 1 Let $f(t, s)=f(t) \in X$ for all $s \in \mathbb{R}$, and

$$
\sum_{j=1}^{p} I_{j}(-\infty) e^{\int_{0}^{t_{j}} a(\tau) \mathrm{d} \tau}<\int_{0}^{T} f(t) e^{\int_{0}^{t} a(\tau) \mathrm{d} \tau} \mathrm{d} t<\sum_{j=1}^{p} I_{j}(+\infty) e^{\int_{0}^{t_{j}} a(\tau) \mathrm{d} \tau}
$$

Then the impulsive problem (1), (2) has at least one solution. Moreover, if for all $s \in \mathbb{R}$, $j=1,2, \ldots, p$, we have

$$
I_{j}(-\infty)<I_{j}(s)<I_{j}(+\infty)
$$

then (7) is also a necessary condition for the existence of a solution. A similar assertion holds true if we switch $I_{j}(-\infty)$ and $I_{j}(+\infty)$ in the above inequalities.

We give the following simple examples, which illustrate our main results.

Example 1 Let us consider the linear periodic problem

$$
\begin{aligned}
& u^{\prime}(t)+u(t) \cos t=f(t), \quad t \in[0,2 \pi] \\
& u(0)=u(2 \pi) .
\end{aligned}
$$

It is well known that (9) has a solution if and only if

$$
\int_{0}^{2 \pi} f(t) e^{\sin t} \mathrm{~d} t=0
$$

The impulse condition

$$
u\left(\pi^{+}\right)=u(\pi)-\arctan u(\pi)
$$

corresponds to $t_{1}=\pi, I_{1}(s)=\arctan s$ and

$$
-\frac{\pi}{2}=I_{1}(-\infty)<I_{1}(s)<I_{1}(+\infty)=\frac{\pi}{2}
$$


It follows from Corollary 1 that the linear problem (9) with nonlinear impulse (11) has a solution if and only if

$$
-\frac{\pi}{2}<\int_{0}^{2 \pi} f(t) e^{\sin t} \mathrm{~d} t<\frac{\pi}{2} .
$$

Comparison of (10) and (12) shows that the problem with impulses has a solution for a wider class of external forces $f$ than the original problem without impulses.

Similarly, applying Corollary 1, we obtain an existence result for the linear problem (9) with impulse condition

$$
u\left(\pi^{+}\right)=u(\pi)-\operatorname{arccot} u(\pi)
$$

if and only if

$$
0<\int_{0}^{2 \pi} f(t) e^{\sin t} \mathrm{~d} t<\pi
$$

Comparison of (10) and (14) shows that the sets of external forces $f$ for which the problem with impulse (13) and the problem without impulse (13) have a solution have an empty intersection.

Example 2 Let us consider the nonlinear periodic problem

$$
\begin{aligned}
& u^{\prime}(t)+u(t) \cos t=\cos t \cdot \arctan u(t), \quad t \in[0,2 \pi] \\
& u(0)=u(2 \pi)
\end{aligned}
$$

with impulses (2). In this case, we have $f_{ \pm}(t)= \pm \frac{\pi}{2} \cos t$ and

$$
\int_{0}^{2 \pi} f_{ \pm}(t) e^{\int_{0}^{t} a(\tau) \mathrm{d} \tau} \mathrm{d} t=0
$$

It follows from Theorem 1 that the nonlinear impulse problem (15), (2) has at least one solution, provided that

$$
\sum_{j=1}^{p} I_{j}(-\infty) e^{\sin t_{j}}<0<\sum_{j=1}^{p} I_{j}(+\infty) e^{\sin t_{j}}
$$

Example 3 Let us consider the nonlinear periodic problem (1) with impulses (2). Assume $I_{j}(+\infty)=+\infty$ for at least one $j \in\{1, \ldots, p\},\left|I_{j}(+\infty)\right|<\infty$ otherwise and, similarly, $I_{k}(-\infty)=$ $-\infty$ for at least one $k \in\{1, \ldots, p\}$ and $\left|I_{k}(-\infty)\right|<-\infty$ otherwise. Then the impulse problem (1), (2) has at least one solution.

The above examples illustrate that suitably chosen impulses may control the forcing term in order to get existence or nonexistence of periodic solutions. 


\section{Some elementary facts}

For the reader's convenience we provide a survey of well-known facts about the linear periodic problem and constant impulses:

(i) (Nonresonance case) Periodic problem

$$
\begin{aligned}
& u^{\prime}(t)+a(t) u(t)=f(t), \quad t \in J, \\
& u(0)=u(T)
\end{aligned}
$$

has a unique solution for all $f$ if and only if $\int_{0}^{T} a(\tau) \mathrm{d} \tau \neq 0$.

This solution is expressed in the following form:

$$
u(t)=\int_{0}^{T} g_{a}(t, s) f(s) \mathrm{d} s
$$

where

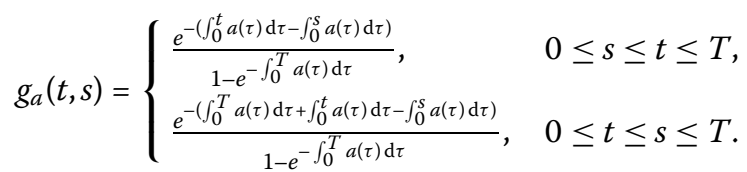

In particular, $\int_{0}^{T} a(\tau) \mathrm{d} \tau>0$ implies $g_{a}(t, s)>0 \forall t, s \in J$.

(ii) (Nonresonance case with impulses) If $\int_{0}^{T} a(\tau) \mathrm{d} \tau \neq 0$, the solution of (16) on $J^{\prime}$ with constant impulses

$$
u\left(t_{j}^{+}\right)=u\left(t_{j}\right)+\theta_{j}, \quad j=1,2, \ldots, p
$$

is given by

$$
u(t)=\int_{0}^{T} g_{a}(t, s) f(s) \mathrm{d} s+\sum_{j=1}^{p} g_{a}\left(t, t_{j}\right) \theta_{j} .
$$

(iii) (Resonance case) If $\int_{0}^{T} a(\tau) \mathrm{d} \tau=0$, then (16) has a solution if and only if

$$
\int_{0}^{T} f(t) e^{\int_{0}^{t} a(\tau) \mathrm{d} \tau} \mathrm{d} t=0
$$

The solution (if it exists) is then not unique. It is given by

$$
u(t)=c e^{-\int_{0}^{t} a(\tau) \mathrm{d} \tau}+e^{-\int_{0}^{t} a(\tau) \mathrm{d} \tau} \int_{0}^{t} f(\tau) e^{\int_{0}^{\tau} a(\sigma) \mathrm{d} \sigma} \mathrm{d} \tau,
$$

where $c \in \mathbb{R}$ is arbitrary.

(iv) (Resonance case with impulses) If $\int_{0}^{T} a(\tau) \mathrm{d} \tau=0$, then (16) on $J^{\prime}$ with impulses (17) has a solution if and only if

$$
\int_{0}^{T} f(t) e^{\int_{0}^{t} a(\tau) \mathrm{d} \tau} \mathrm{d} t+\sum_{i=1}^{p} e^{\int_{j}^{t_{j}} a(\tau) \mathrm{d} \tau} \theta_{j}=0 .
$$


The solution (if it exists) is not unique. It is given by

$$
u(t)=c e^{-\int_{0}^{t} a(\tau) \mathrm{d} \tau}+e^{-\int_{0}^{t} a(\tau) \mathrm{d} \tau}\left[\int_{0}^{t} f(\tau) e^{\int_{0}^{\tau} a(\sigma) \mathrm{d} \sigma} \mathrm{d} \tau+\sum_{i=1}^{p} e^{\int_{0}^{t_{j}} a(\tau) \mathrm{d} \tau} \theta_{j}\right]
$$

where $c \in \mathbb{R}$ is arbitrary.

The properties (i)-(iv) follow from the variation of constants formula.

\section{Proofs}

Fix $\delta>0$ and set $a_{\delta}(t):=a(t)+\delta$. Then $a_{\delta} \in X$ and $\int_{0}^{T} a_{\delta}(t) \mathrm{d} t=T \delta>0$. The periodic problem (1) with impulses (2) can be written in an equivalent form:

$$
\begin{aligned}
& u^{\prime}(t)+a_{\delta}(t) u(t)=\delta u(t)+f(t, u(t)), \quad t \in J^{\prime}, \\
& u(0)=u(T), \quad u\left(t_{j}^{+}\right)=u\left(t_{j}\right)-I_{j}\left(u\left(t_{j}\right)\right), \quad j=1,2, \ldots, p .
\end{aligned}
$$

We define operator $F: X \rightarrow X$ as follows:

$$
F(u)(t):=\int_{0}^{T} g_{a_{\delta}}(t, s)[\delta u(s)+f(s, u(s))] \mathrm{d} s-\sum_{j=1}^{p} g_{a_{\delta}}\left(t, t_{j}\right) I_{j}\left(u\left(t_{j}\right)\right) .
$$

According to (ii), $u \in X$ is a solution of (18) (and hence also of (1), (2)) if and only if $u$ is a fixed point of $F$, i.e.,

$$
u=F(u)
$$

The operator $F$ is compact. Indeed, let $M \subset X$ be a bounded set. Then it follows from the definition of $F$ that the family of functions $F(M)$ is equicontinuous in $B C\left(J^{\prime}\right)$ and for each $t \in J^{\prime}$ the set $\{u(t): u \in F(M)\}$ is bounded in $\mathbb{R}$. It follows from Arzela-Ascoli's theorem (see [20, Theorem 1.2.13]) that $F(M)$ is relatively compact in $B C\left(J^{\prime}\right)$ and hence also in $X$. Since $F$ is clearly continuous, compactness of $F$ follows.

The following a priori estimate is the key to the proof of Theorem 1.

Lemma 1 There exists $K>0$ such that for all $\lambda \in[0,1]$ and $u \in X$ satisfying $u=\lambda F(u)$ we have $\|u\|<K$.

Proof Assume via contradiction that there exist $u_{n} \in X, \lambda_{n} \in[0,1](n \in \mathbb{N})$ such that $\left\|u_{n}\right\| \geq n$ and

$$
u_{n}=\lambda_{n} F\left(u_{n}\right) .
$$

This is equivalent to

$$
\begin{aligned}
& u_{n}^{\prime}(t)+a_{\delta}(t) u_{n}(t)=\lambda_{n}\left(\delta u_{n}(t)+f\left(t, u_{n}(t)\right)\right), \quad t \in J^{\prime}, \\
& u_{n}(0)=u_{n}(T), \quad u_{n}\left(t_{j}^{+}\right)=u_{n}\left(t_{j}\right)-\lambda_{n} I_{j}\left(u_{n}\left(t_{j}\right)\right), \quad j=1,2, \ldots, p .
\end{aligned}
$$


Set $v_{n}:=\frac{u_{n}}{\left\|u_{n}\right\|}$. Then $v_{n} \in X ;\left\|v_{n}\right\|=1$, satisfy

$$
\begin{aligned}
& v_{n}^{\prime}(t)+a_{\delta}(t) v_{n}(t)=\lambda_{n}\left(\delta v_{n}(t)+\frac{f\left(t, u_{n}(t)\right)}{\left\|u_{n}\right\|}\right), \quad t \in J^{\prime}, \\
& v_{n}(0)=v_{n}(T), \quad v_{n}\left(t_{j}^{+}\right)=v_{n}\left(t_{j}\right)-\lambda_{n} \frac{I_{j}\left(u_{n}\left(t_{j}\right)\right)}{\left\|u_{n}\right\|}, \quad j=1,2, \ldots, p .
\end{aligned}
$$

This is equivalent to the integral equation

$$
v_{n}(t)=\lambda_{n} \int_{0}^{T} g_{a_{\delta}}(t, s)\left[\delta v_{n}(s)+\frac{f\left(s, u_{n}(s)\right)}{\left\|u_{n}\right\|}\right] \mathrm{d} s-\lambda_{n} \sum_{j=1}^{p} g_{a_{\delta}}\left(t, t_{j}\right) \frac{I_{j}\left(u_{n}\left(t_{j}\right)\right)}{\left\|u_{n}\right\|} .
$$

Since $\left|v_{n}(s)\right| \leq 1, \frac{f\left(s, u_{n}(s)\right)}{\left\|u_{n}\right\|} \rightarrow 0$, uniformly with respect to $s \in[0, T], \frac{I_{j}\left(u_{n}\left(t_{j}\right)\right)}{\left\|u_{n}\right\|} \rightarrow 0, j=$ $1,2, \ldots, p$, by similar argument as above, the family $\left\{v_{n}\right\}$ is equicontinuous in $B C\left(J^{\prime}\right)$ and for each $t \in J^{\prime}$ the set $\left\{v_{n}(t)\right\}$ is bounded. It follows from Arzela-Ascoli's theorem that $\left\{v_{n}\right\}$ is relatively compact in $B C\left(J^{\prime}\right)$ and hence also in $X$.

Passing to subsequences, if necessary, we may assume that there exist $v \in X$ and $\lambda \in[0,1]$ such that $v_{n} \rightarrow v$ in $X, \lambda_{n} \rightarrow \lambda$. Taking the limit for $n \rightarrow \infty$ in (20) we get

$$
v(t)=\lambda \int_{0}^{T} g_{a_{\delta}}(t, s) \delta v(s) \mathrm{d} s,
$$

or equivalently

$$
\begin{aligned}
& v^{\prime}(t)+a_{\delta}(t) v(t)=\lambda \delta v(t), \quad t \in J, \\
& v(0)=v(T) .
\end{aligned}
$$

If $\lambda \neq 1$ then (21) has only a trivial solution (see (i)). But this contradicts $\|v\|=1$ ! Hence, $\lambda=1$ and $\nu \in X$ solves

$$
\begin{aligned}
& v^{\prime}(t)+a(t) v(t)=0, \quad t \in J, \\
& v(0)=v(T),
\end{aligned}
$$

i.e.,

$$
v(t)= \pm c \cdot e^{-\int_{0}^{t} a(\tau) \mathrm{d} \tau}
$$

where $c>0$ is such that $\|v\|=1$.

Consider first the case $v(t)=+c \cdot e^{-\int_{0}^{t} a(\tau) \mathrm{d} \tau}>0$ in $J$. Since $v_{n} \rightarrow v$ in $X$ (i.e., uniformly in $J$ ) we have $u_{n}(t) \rightarrow+\infty$ as $n \rightarrow \infty$ uniformly in $J$. Multiply the equation in (19) by $e^{\int_{0}^{t} a(\tau) \mathrm{d} \tau}$, integrate over $J$ and perform the integration by parts in the first integral to get

$$
\begin{aligned}
& \sum_{j=0}^{p}\left[u_{n}(t) e^{\int_{0}^{t} a(\tau) \mathrm{d} \tau}\right]_{t_{j}}^{t_{j+1}} \underbrace{\int_{0}^{T} a(t) e^{\int_{0}^{t} a(\tau) \mathrm{d} \tau} u_{n}(t) \mathrm{d} t+\int_{0}^{T} a(t) u_{n}(t) e^{\int_{0}^{t} a(\tau) \mathrm{d} \tau} \mathrm{d} t}_{0} \\
& \quad+\delta\left(1-\lambda_{n}\right) \int_{0}^{T} u_{n}(t) e^{\int_{0}^{t} a(\tau) \mathrm{d} \tau} \mathrm{d} t=\int_{0}^{T} f\left(t, u_{n}(t)\right) e^{\int_{0}^{t} a(\tau) \mathrm{d} \tau} \mathrm{d} t .
\end{aligned}
$$


Since

$$
\begin{aligned}
\sum_{j=0}^{p}\left[u_{n}(t) e^{\int_{0}^{t} a(\tau) \mathrm{d} \tau}\right]_{t_{j}}^{t_{j+1}}= & \sum_{j=0}^{p}\left[u_{n}\left(t_{j+1}\right) e^{\int_{0}^{t_{j+1}} a(\tau) \mathrm{d} \tau}-u_{n}\left(t_{j}^{+}\right) e^{\int_{0}^{t_{j}} a(\tau) \mathrm{d} \tau}\right] \\
= & \underbrace{u_{n}(T) e^{\int_{0}^{T} a(\tau) \mathrm{d} \tau}-u_{n}(0)}_{0}+\underbrace{\left[u_{n}\left(t_{1}\right)-u_{n}\left(t_{1}^{+}\right)\right]}_{I_{1}\left(u_{n}\left(t_{1}\right)\right)} e^{\int_{0}^{t_{1}} a(\tau) \mathrm{d} \tau}+\cdots \\
& +\underbrace{\left[u_{n}\left(t_{p}\right)-u_{n}\left(t_{p}^{+}\right)\right]}_{I_{p}\left(u_{n}\left(t_{p}\right)\right)} e^{\int_{0}^{t_{p}} a(\tau) \mathrm{d} \tau}=\sum_{j=1}^{p} I_{j}\left(u_{n}\left(t_{j}\right)\right) e^{\int_{0}^{t_{j}} a(\tau) \mathrm{d} \tau},
\end{aligned}
$$

we arrive at

$$
\sum_{j=1}^{p} I_{j}\left(u_{n}\left(t_{j}\right)\right) e^{\int_{0}^{t_{j}} a(\tau) \mathrm{d} \tau}+\delta\left(1-\lambda_{n}\right) \int_{0}^{T} u_{n}(t) e^{\int_{0}^{t} a(\tau) \mathrm{d} \tau} \mathrm{d} t=\int_{0}^{T} f\left(t, u_{n}(t)\right) e^{\int_{0}^{t} a(\tau) \mathrm{d} \tau} \mathrm{d} t
$$

Recall that $u_{n}(t) \rightarrow+\infty$ uniformly in $J$. In particular, we have also $u_{n}\left(t_{j}\right) \rightarrow+\infty, j=$ $1,2, \ldots, p$. These facts together with (22), boundedness of $f$, and the Lebesgue dominated convergence theorem yield

$$
\int_{0}^{T} f_{+}(t) e^{\int_{0}^{t} a(\tau) \mathrm{d} \tau} \mathrm{d} t \geq \sum_{j=1}^{p} I_{j}(+\infty) e^{\int_{0}^{t_{j}} a(\tau) \mathrm{d} \tau}
$$

a contradiction with (3).

Similarly, if $v(t)=-c \cdot e^{-\int_{0}^{t} a(\tau) \mathrm{d} \tau}<0$ in $J$, we arrive at a contradiction with (4).

Compactness of $F$, Lemma 1 and the Schaeffer fixed point theorem (see [20, Example 5.8.4]) imply that (1), (2) has a solution provided that (3) and (4) hold true. Choosing $\delta<0$ at the beginning of the proof, we get the existence result provided that (5) and (6) hold.

The proof of Theorem 1 is finished.

Let $f=f(t, s)$ be independent of $s$, that is, $f(t, s)=f(t), t \in J, s \in \mathbb{R}$. Then $f(t)=f_{+}(t)=f_{-}(t)$ and (3), (4) reduces to (7). Hence the linear problem (16) with nonlinear impulses (2) has a solution provided that $(7)$ holds.

We prove that condition (7) is also necessary under assumption (8). Indeed, let $u$ be a solution of (16), (2). Multiply the equation in (16) by $e^{\int_{0}^{t} a(\tau) \mathrm{d} \tau}$ and integrate over $J$ with respect to $t$ :

$$
\int_{0}^{T} u^{\prime}(t) e^{\int_{0}^{t} a(\tau) \mathrm{d} \tau} \mathrm{d} t+\int_{0}^{T} a(t) u(t) e^{\int_{0}^{t} a(\tau) \mathrm{d} \tau} \mathrm{d} t=\int_{0}^{T} f(t) e^{\int_{0}^{t} a(\tau) \mathrm{d} \tau} \mathrm{d} t
$$

Integrating by parts the first integral we get

$$
\begin{aligned}
& \sum_{j=0}^{p}\left[u(t) e^{\int_{0}^{t} a(\tau) \mathrm{d} \tau}\right]_{t_{j}}^{t_{j+1}} \underbrace{\int_{0}^{T} a(t) e^{\int_{0}^{t} a(\tau) \mathrm{d} \tau} u(t) \mathrm{d} t+\int_{0}^{T} a(t) u(t) e^{\int_{0}^{t} a(\tau) \mathrm{d} \tau} \mathrm{d} t}_{0} \\
& \quad=\int_{0}^{T} f(t) e^{\int_{0}^{t} a(\tau) \mathrm{d} \tau} \mathrm{d} t .
\end{aligned}
$$


Evaluating the sum on the left-hand side as above, we arrive at

$$
\sum_{j=1}^{p} I_{j}(u(t)) e^{\int_{0}^{t_{j}} a(\tau) \mathrm{d} \tau}=\int_{0}^{T} f(t) e^{\int_{0}^{t} a(\tau) \mathrm{d} \tau} \mathrm{d} t .
$$

Inequality (7) follows immediately from (23) provided that (8) holds true. The proof of Corollary 1 is finished.

\section{Competing interests}

The authors declare that they have no competing interests.

\section{Authors' contributions}

Both authors read and approved the final manuscript.

\section{Author details}

${ }^{1}$ Department of Mathematics, University of West Bohemia, Univerzitní 22, Plzeň, 306 14, Czech Republic. ${ }^{2}$ NTIS, University of West Bohemia, Univerzitní 22, Plzeň, 306 14, Czech Republic.

\section{Acknowledgements}

This research was supported by the Grant 13-00863S of the Grant Agency of Czech Republic and by the European Regional Development Fund (ERDF), project 'NTIS - New Technologies for the Information Society', European Centre of Excellence, CZ.1.05/1.1.00/02.0090.

Received: 2 May 2014 Accepted: 19 July 2014 Published online: 26 September 2014

\section{References}

1. Lakshmikantham, V, Bainov, DD, Simeonov, PS: Theory of Impulsive Differential Equations. World Scientific, Cambridge (1989)

2. Bainov, DD, Simeonov, PS: Impulsive Differential Equations: Periodic Solutions and Applications. Longman, Harlow (1993)

3. Samoilenko, AM, Perestyuk, NA: Impulsive Differential Equations. World Scientific, Singapore (1995)

4. Boichuk, AA, Samoilenko, AM: Generalized Inverse Operators and Fredholm Boundary-Value Problems. VSP, Utrecht (2004)

5. Agarwal, RP, Franco, D, O'Regan, D: Singular boundary value problems for first and second order impulsive differential equations. Aequ. Math. 69, 83-96 (2005)

6. Qian, D, Li, X: Periodic solutions for ordinary differential equations with sublinear impulsive effects. J. Math. Anal. Appl. 303, 288-303 (2005)

7. Cai, G, Du, Z, Ge, W: Periodic boundary value problem for first order impulsive differential equation at resonance. Rocky Mt. J. Math. 37(1), 67-77 (2007). doi:10.1216/rmjm/1181069320

8. Franco, D, Nieto, Jj: First-order impulsive ordinary differential equations with anti-periodic and nonlinear boundary conditions. Nonlinear Anal. 42, 163-173 (2000)

9. Nieto, JJ: Impulsive resonance periodic problems of first order. Appl. Math. Lett. 15, 489-493 (2002)

10. Nieto, JJ: Periodic boundary value problems for first-order impulsive ordinary differential equations. Nonlinear Anal. 51, 1223-1232 (2002)

11. Li, J, Nieto, JJ, Shen, J: Impulsive periodic boundary value problems of first-order differential equations. J. Math. Anal. Appl. 325(1), 226-236 (2007)

12. Chu, J, Nieto, JJ: Impulsive periodic solutions of first-order singular differential equations. Bull. Lond. Math. Soc. 40(1), 143-150 (2008)

13. Carter, TE: Optimal impulsive space trajectories based on linear equations. J. Optim. Theory Appl. 70, 277-297 (1991)

14. Carter, TE: Necessary and sufficient conditions for optimal impulsive rendezvous with linear equations of motion. Dyn. Control 10, 219-227 (2000)

15. Liu, X, Willms, AR: Impulsive controllability of linear dynamical systems with applications to maneuvers of spacecraft. Math. Probl. Eng. 2, 277-299 (1996)

16. Prado, AFBA: Bi-impulsive control to build a satellite constellation. Nonlinear Dyn. Syst. Theory 5, 169-175 (2005)

17. Landesman, EM, Lazer, AC: Nonlinear perturbations of linear elliptic boundary value problems at resonance. J. Math. Mech. 19, 609-623 (1970)

18. Drábek, P, Langerová, M: Quasilinear boundary value problem with impulses: variational approach to resonance problem. Bound. Value Probl. 2014, 64 (2014)

19. Drábek, P, Langerová, M: On the second order equations with nonlinear impulses - Fredholm alternative type results. Topol. Methods Nonlinear Anal. (in press)

20. Drábek, P, Milota, J: Methods of Nonlinear Analysis: Applications to Differential Equations, 2nd edn. Springer, Basel (2013)

doi:10.1186/s13661-014-0186-3

Cite this article as: Drábek and Langerová: First order periodic problem at resonance with nonlinear impulses.

Boundary Value Problems 2014 2014:186. 